\title{
Identificación melancólica y constitución de la identidad de género masculina. Aportes del psicoanálisis a los estudios contemporáneos de género
}

\author{
Melancholic identification and male gender identity \\ constitution. Psychoanalytic contributions to \\ contemporary gender studies
}

Ariel Martinez ${ }^{1}$

Resumen

Desde la confluencia entre el psicoanálisis y los estudios de género, este trabajo intenta delimitar algunos aspectos de la teoría intersubjetiva de Jessica Benjamin en relación con el proceso de constitución de la identidad de género masculina hegemónica. A partir de allí, se presentan parte de las consideraciones conceptuales que Judith Butler realiza en relación con la melancolía postulada por Freud, y su conexión con la conformación subjetiva del género para delimitar el modo en que se conceptualiza la constitución melancólica de la identidad de género. Se concluye que, a partir del entrecruzamiento de ambas autoras, se despliega una plataforma analítica que permite avanzar hacia nuevas articulaciones que delimitan, nunca de manera definitiva, una posible explicación psicodinámica de la constitución de la identidad de género a partir del mecanismo de identificación, de modo tal que se incluyen niveles de análisis que van más allá de mecanismos individuales de funcionamiento.

Palabras Clave: Identificación, Género, Identidad, Heterosexualidad.

1 Ariel Martínez, Centro Interdisciplinario de Investigaciones en Género (CINIG) de la Universidad Nacional de La Plata / Comisión de Investigaciones Científicas (CIC) de la Provincia de Buenos Aires. Profesor de Psicología y Licenciado en Psicología, Facultad de Psicología, Universidad Nacional de La Plata, La Plata. Argentina. amartinez@psico.unlp.edu.ar. 


\begin{abstract}
From the intersection between psychonalysis and genders studies, this paper tries to delimit some aspects of Jessica Benjamin's intersubjectivity theory in connection with the process of constitution of the hegemonic male gender identity. From that point, Judith Butler's conceptual considerations are presented in relation to melancholy, as postulated by Freud, and its connection with the subjective conformation of gender, in order to circumscribe the way in which the melancholic constitution of the gender identity is conceptualized. It is concluded that from the intertwining of both authors, an analytic platform is displayed allowing to move forward to new articulations that define, never in a definite way, a plausible psychodynamic explanation of gender identity constitution from the identification mechanism so as to incorporates levels of analysis that go beyond individual mechanism of operation.
\end{abstract}

Key words: Identification, Gender, Identity, Heterosexuality.

Planteamiento del tema a abordar

En las últimas décadas ha ido adquiriendo cada vez mayor relevancia el debate respecto a la diferencia entre varones y mujeres. La categoría de género al interior de las Ciencias Sociales se ha tornado crucial para el análisis de las diferencias entre los sexos (Lamas, 1986). En términos descriptivos, se puede entender por género:

...la red de creencias, rasgos de personalidad, actitudes, valores, conductas y actividades [construidas y asignadas socialmente] que diferencian a mujeres y a hombres. Tal diferenciación (...) no solo produce diferencias entre los géneros femenino y masculino, sino que a la vez, estas diferencias implican desigualdades y jerarquías entre ambos. (Burin \& Meler, 1998, p.20). 
En este sentido, los estudios de género denuncian la lógica binaria al tiempo que visibilizan la jerarquía intrínseca a tal ordenamiento ligado a largos procesos histórico-sociales. Las relaciones entre varones y mujeres no son abordadas en términos naturales sino como resultado de un proceso histórico que emerge y se reproduce por estructuras sociales patriarcales. En este contexto, los recientes análisis filosóficos feministas apelan a la teoría psicoanalítica, centrándose fundamentalmente en la categoría de identificación para dar cuenta del modo diferencial en que se constituyen las identidades de género a nivel subjetivo.

Robert Stoller (1968), ha sido el primero en diferenciar los roles de género -conjunto de conductas socialmente atribuidas a mujeres y a varones- de la identidad de género-sentimiento de pertenencia al conjunto varón o mujer que se establece precozmente, antes del conocimiento que cada niño/a tiene de la diferencia sexual anatómica y el papel de los genitales en la reproducción-. Es decir, el sentimiento que el propio niño/a tiene de ser varón o mujer (Lamas, 1986). En palabras de Emilce Dio Bleichmar (1992), se trata del:

...conjunto de prescripciones y prohibiciones para el ejercicio de una conducta, así como un sentimiento del ser que se reconoce (femenino o masculino) por desempeñar las actividades y conductas propias de su condición, y es reconocido por los otros en tanto se ajusta a ese desempeño esperado (p.135).

En esta línea, el presente trabajo intenta delimitar algunas de las coordenadas culturales y políticas que subyacen a la constitución de las identidades de género. Para ello, se presentan algunos aspectos de la teoría intersubjetiva de Jessica Benjamin en relación con el proceso de constitución de la identidad de género masculina en sus modalidades hegemónicas. Luego, se presentan parte de las consideraciones filosóficas que Judith Butler realiza sobre el mecanismo melancólico, postulado por Freud, y su conexión con la conformación subjetiva del género, para luego delimitar el modo en que conceptualiza la constitución melancólica de la identidad de género. Se enfatizan aquellos desarrollos de la autora que 
giran en torno a la categoría de identificación, ligada a la pérdida sufrida durante el proceso de formación de la identidad.

Finalmente, se despliega una plataforma analítica que se propone delimitar, nunca de manera definitiva, aspectos que conforman una posible explicación psicodinámica de la constitución de la identidad de género a partir del mecanismo de identificación. Sin dejar de tener en cuenta las condiciones culturales que configuran el contexto, se incluyen niveles de análisis que van más allá de mecanismos individuales de funcionamiento.

\section{Desarrollo Teórico}

Jessica Benjamin (1996), psicoanalista norteamericana, aborda el estudio del desarrollo del sí-mismo en términos de dominación-sumisión a través de una teoría intersubjetiva, lo que le permite entramar la variable género en el proceso de constitución subjetiva. La autora, ubicada en la línea del psicoanálisis de las relaciones objetales, se centra en los intentos sistemáticos por comprender las relaciones de género a partir de la posibilidad de un reconocimiento mutuo en términos dialécticos. A partir de allí se desprende su tesis central: la dominación masculina y la sumisión femenina son resultado de una ruptura de la tensión necesaria entre la autoafirmación del si-mismo y el reconocimiento del otro como un sujeto igual. El mantenimiento de esta tensión permite al si-mismo y al otro encontrarse como sujetos iguales.

La relación entre la autoafirmación y el reconocimiento es concebida por Benjamin (1996) en términos de una paradoja, pues en el momento mismo en que se comprende la propia independencia, se depende de otro que la reconozca. En este sentido Benjamin argumenta que es necesario resistir a la resolución de esta paradoja, pues la incapacidad de sostenerla implica la caída del reconocimiento, por tanto la dominación y la sumisión.

Las lecturas que realizan Nancy Chodorow (1974, 1978) y, posteriormente, Benjamin $(1996,1997)$ del modelo edípico freudiano clásico, permiten comprender los vínculos existentes entre tal modelo teórico y los modos en que al interior de una organización social patriarcal 
se ordenan los vínculos. En esta línea, Benjamin (1996) critica el supuesto freudiano de que el si-mismo debe ser postulado en oposición a un otro/ objeto. Benjamin no conceptualiza esta estructura sujeto-objeto -que subyace a la conformación del si-mismo- como una condición natural, sino como el producto del fracaso de las relaciones intersubjetivas. Sin embargo, en tanto se trata de un modelo patriarcal, refleja las condiciones de emergencia de la identidad masculina a partir del repudio y la exclusión de lo otro/objeto ligado a lo femenino.

La teoría intersubjetiva de Jessica Benjamin (1996, 1997, 1998) ofrece los elementos conceptuales necesarios para comprender el modo en que la identidad de género masculina hegemónica, que emerge a través de condiciones patriarcales legitimantes, se constituye a partir de un repudio inaugural de lo femenino que captura, indefectiblemente, a tal identidad en un entramado conflictivo. En este contexto, el supuesto freudiano de un deseo fundamental de la negación del otro refiere solo a las particularidades que adquiere la masculinidad en un contexto cultural que impone y exige identificaciones excluyentes y direcciones específicas de deseo (Butler, 1990) en la constitución identitaria.

La ficción de la simbiosis y el problema de la desidentificación

Freud ha referido a la vivencia de satisfacción, producto del encuentro entre la boca del infans y el pecho de la madre, como un primer movimiento que inaugura un sentimiento de completud. En palabras de Julia Kristeva (2009):

...se trataría de la unión íntima del Yo con el mundo circundante, sentida como una certeza absoluta de satisfacción, de seguridad (...), y que remite a la vivencia del lactante que aún no ha establecido fronteras entre su Yo y el cuerpo materno. (...) Una ósmosis jubilosa del sujeto en la carne común de un 'aún-no uno mismo', tragado por un aún-no mundo (p.19).

Jessica Benjamin (1997) retoma estas ideas y las vincula con el misterio de la maternidad. Expone el modo en que el temor al poder materno, en la base del temor inconscientemente arraigado que las mujeres 
despiertan en los varones (Rich, 1986), se pone al servicio de una amplia gama de propósitos.

Tal como han señalado varias pensadoras -entre ellas Kaja Silverman (1988) y Hortense Spillers (1987)-, el psicoanálisis ha arrojado un modelo que naturaliza la constitución subjetiva de los/as niños/as signada por una profunda dependencia inicial a la figura de la madre (Driver, 2005). Sin embargo, este aterrador sentimiento oceánico generado por las profundidades maternas encuentra, a nivel teórico, su antídoto: un padre omnipotente que posteriormente lleva a cabo la separación y quebranta esa mónada compacta y asfixiante que no permite la emergencia de un sujeto en clave fálica. Tal como afirma Kristeva (2009), la figura del padre consigue despertar al niño de la hipnosis de lo materno arcaico a la cual el bebé parece sucumbir fácilmente.

A criterio de Benjamin (1997), tal unidad originaria es una construcción teórica del psicoanálisis para explicar el miedo a la regresión hacia lo primario y desubjetivante. A través de estos supuestos teóricos que giran en torno a la dependencia, la madre perdida es recodificada retroactivamente como un otro peligroso.

En este contexto conceptual, Jessica Benjamin (1996) critica el supuesto freudiano de que el objetivo normativo del desarrollo del si-mismo sea la separación, la cual supone una transición desde un estado de conexión o no diferenciación hacia un estado de separación o independencia. El supuesto es que para constituirse en sujetos es necesario romper con un estado de inmediatez o no diferenciación con la madre a través de la internalización de la autoridad paterna. Jessica Benjamin (1996), a partir de los aportes de Nancy Chodorow (1978), sostiene que el desarrollo de las identidades de género masculinas y femeninas, y la dominación masculina y la subordinación femenina, son producidos, al menos en parte, por el lugar que la cultura patriarcal asigna a las mujeres en la crianza de los nińos.

Dice Benjamin (1996):

... como en casi todas partes las mujeres han sido las cuidadoras primarias de [los/as niños/as pequeños/as], tanto los niños como las 
niñas se han diferenciado en relación con una mujer. Cuando consideramos el curso típico de la diferenciación masculina, vemos de inmediato que crea una dificultad especial para los varones. Todos los nińos se identifican con su primer ser querido, pero los varones deben disolver esta identificación y definirse como el sexo diferente. Al principio [los/as niños/as] se sienten semejantes a sus madres. Pero los varones descubren que no pueden llegar a ser como ella; solo pueden tenerla. Este descubrimiento conduce a una ruptura de la identificación, que las niñas no tienen que sufrir. Los varones afirman su masculinidad negando la identificación o unidad original con sus madres (pp.98-99).

En efecto, los niños forman su identidad de género por desidentificación de una mujer y, solo secundariamente, por la identificación con el padre. Benjamin (1996) continúa:

El varón desarrolla su género y su identidad estableciendo una discontinuidad y una diferencia respecto de la persona a la que está más apegado. Este proceso de desidentificación explica el repudio a la madre que subtiende la formación convencional de la identidad masculina. (pp.99-100).

El desarrollo del sí-mismo del varón es descrito con precisión como un proceso que supone represión, dominación, y negación del otro -materno en un primer momento, de las mujeres posteriormente. Es necesario, entonces, disolver la identificación con la madre para ser confirmado como un sujeto/masculino. El logro de tal inteligibilidad ligada a una "exitosa" salida del Edipo supone el hecho de no reconocer a la madre como un sujeto igual, de modo que una actitud objetivante viene a remplazar las interacciones afectivas anteriores (Weir, 1992), pre-edípicas, con la madre, en donde aún, al menos en la teoría de Benjamin, era posible el reconocimiento mutuo.

En suma, Benjamin (1996) deja en claro que la identidad masculina hegemónica resulta de un proceso de separación reactiva que trae consigo la dominación de la madre, quien es relegada al campo de los objetos. De aquí en más, Benjamin (1996) da por sentado el mismo destino para todas las mujeres, pues en tanto mujeres forman parte del género oprimido culturalmente. Esta vinculación entre madre y mujeres, como recurso 
para explicar el modo en que se generaliza el temor y la dominación, no aparece plenamente explorada. Es posible pensar que en las argumentaciones de Benjamin se filtra lo que Ana María Fernández (2010) ha conceptualizado como el mito mujer $=$ madre. Sea como fuere, es posible pensar que las sociedades en su conjunto, al menos las occidentales, organizan sus universos de significaciones en torno a este mito que instala la maternidad como destino normativo de las mujeres. Aún así, tal ecuación opera fluidamente en la cultura, lo que no desacredita el modelo teórico de constitución de la identidad de género masculina, al que Benjamin (1996) sitúa claramente en el contexto de un ordenamiento cultural de los géneros que naturaliza tal mito.

El modelo presentado permite a Benjamin $(1996,1997)$ pensar una posible relación entre la modalidad específica en que se constituye la identidad masculina hegemónica y la subordinación de las mujeres como una defensa compensatoria de la dependencia y envidia inicial en relación al aterrador poder materno de engendrar. El desprecio naturalizado, inconscientemente arraigado, hacia las mujeres es un efecto de lo que Benjamin (1997) denomina como "transferencia del poder al padre" - "solución paterna” en términos de Michel Tort (2008)-, única vía a través de la cual el nińo puede liberarse de la omnipotencia de la madre pre-edípica. En este sentido, para Benjamin (1996), la figura de la mujer/madre trae consigo una fuerte carga regresiva que pone en peligro los límites de la identidad de género masculina -altamente rígidos por constituirse reactivamente-.

Por otra parte, tal como señala Silvia Bleichmar, el concepto de identificación ha ido adquiriendo una importancia capital en el psicoanálisis en tanto "operación fundamental que genera las condiciones para instituir la subjetividad" (Bleichmar, 1995, p. 201). Si se parte de la idea de que la identificación constituye subjetividad, entonces el intento del niño por rechazar esa primera identificación con la madre será fallida. Las consecuencias psíquicas de lo femenino en tanto primer modelo identificatorio (Aulagnier, 2004) no pueden ser suprimidas. Por tanto, el rechazo a la madre implica el rechazo y la negación de un segmento de la propia vida fantasmática del niño, un intento incesante de sepultar aquellos aspectos ligados culturalmente a la feminidad que deben ser repudiados a cada instante para el logro continuo de la masculinidad hegemónica en tanto identidad rígida, 
coherente, monolítica. De este modo la identidad masculina hegemónica constituida inicialmente por el repudio necesita apelar a tal rechazo en todo momento en que se ve amenazada. Se trata de evitar caer por fuera de la inteligibilidad cultural, cifrada en clave patriarcal. Butler (1990a) permite pensar el modo en que la identidad de género no se constituye en un momento puntual, de una vez y para siempre, sino a cada instante.

Para Benjamin, el contexto de una cultura patriarcal equilibra la economía psíquica de la subjetividad masculina hegemónica a través de un temor inconsciente y un consecuente repudio hacia las mujeres. "Este repudio envuelve a la psique en procesos proyectivos (...) que intensifican el miedo a la omnipotencia del otro, así como la necesidad de retaliar mediante la afirmación de la propia omnipotencia” (Benjamin, 1997, p.114). La tensión se quiebra y "lo otro", lugar en el que la mujer es colocada, se torna depositario de las proyecciones masivas que al mismo tiempo conforman defensivamente un dique de contención a los límites de la propia identidad de género masculina altamente rigidizada.

En la misma línea, Jane Flax (1990) destaca el modo en que Freud ubica a la madre como primer otro significativo durante la fase pre-edípica. Sin embargo, posteriormente, el valor estructurante que el Edipo imprime al desarrollo de la identidad masculina hegemónica en la subjetividad del nińo opera mediante un proceso defensivo contra las fantasías instaladas culturalmente en torno a la feminidad (Rich, 1985).

Identidad de género y diferencia sexual en el pensamiento de Jessica Benjamin

A criterio de Jessica Benjamin (1997), el significado de la diferencia sexual ha comenzado a cambiar al interior de algunas corrientes del psicoanálisis contemporáneo. Si bien la tendencia naturalizante detectada en el pensamiento de Freud se solapa de manera sutil, actualmente la diferencia sexual ya no se considera producto de hechos anatómicos. Por otra parte, la autora es sensible a las críticas que ciertos sectores del feminismo contemporáneo han esgrimido en torno a la categoría de identidad. De este modo, toma el problema de la identidad como un organizador central 
a partir del cual propone un modelo de desarrollo de los géneros centrado en la lógica de la identificación, en tanto proceso psíquico interno. Tal proceso se presenta como uno de los conceptos psicoanalíticos privilegiados para pensar la conformación de la subjetividad, en general, y del anclaje psíquico del género, en particular, desde un punto de vista intersubjetivo articulado con la problematización de la diferencia sexual.

Para explicar el modo en que se organiza la subjetividad del ser humano antes de su paso por el Edipo, Benjamin (1997) retoma las conceptualizaciones freudianas en relación con la identificación. Freud (1921) dice:

Contemporáneamente a esta identificación con el padre, y quizás antes, el varoncito emprende una cabal investidura de objeto de la madre (...) Muestra entonces dos lazos psicológicamente diversos: con la madre, una directa investidura sexual de objeto; con el padre, una identificación que lo toma por modelo (...) Es fácil expresar en una fórmula el distingo entre una identificación de este tipo con el padre y una elección de objeto que recaiga sobre él. En el primer caso el padre es lo que uno querría ser; en el segundo, lo que uno querría tener. La diferencia depende, entonces, de que la ligazón recaiga en el sujeto o en el objeto del yo (pp. 99-100).

A partir de este aporte es posible deslindar el modo en que la lógica de la identidad articulada por el Edipo freudiano se organiza en torno a lo que Benjamin ha dado en llamar eje igualdad-diferencia. Es así que, bajo este eje, la identificación tiende hacia lo igual, al tiempo que el abismo de la diferencia constituye las fronteras entre las identidades opuestas (Campbell, 1997). Reconocer tal diferencia implica delimitar el conjunto de representaciones, actitudes, sentimientos que quedan por fuera del modelo social incorporado a través de la identificación, para luego repudiarlo y circunscribirlo a aquello que uno no es, lo que uno nunca fue, incluso lo que uno no podrá ser jamás. Esta concepción de LA diferencia, singular, y la concomitante direccionalidad impuesta a la identificación, conduce a las identidades genéricas por la vía de la coherencia, la 
inevitabilidad, singularidad y uniformidad. En este contexto conceptual Benjamin (1997) afirma que la identidad opera como un destino.

En esta línea, en donde solo se puede ser lo igual y tener la diferencia, la autora se inclina por generar un relato contemporáneo del desarrollo del género por fuera del modelo edípico. Para ello privilegia la categoría de identificación, pero no LA identificación que entreteje las identidades rígidas y normativas que arroja el Edipo dicotómico y heterosexual freudiano, sino que deslinda la posibilidad de concebir identidades inestables y múltiples (Gergen, 1992; Reinharz, 1992; Thompson, 1992; Young, 1992) que permiten interrogar la diferencia sexual al desenmarcarla de la estructura edípica convencional.

En este sentido, Benjamin (1997) revaloriza la identificación con la diferencia. A su criterio, tal afirmación encierra una paradoja en relación con los criterios que ordenan las identidades al interior del eje igualdaddiferencia. La identificación con la diferencia atraviesa la línea demarcatoria de lo idéntico, de modo que incorpora algo de lo diferente en la formación del sí-mismo. Simultáneamente, Benjamin (1997) retoma a Fast (1990), quien sitúa la bisexualidad freudiana en el plano identificatorio. Ya desde las interacciones pre-edípicas las representaciones del sí mismo se sostienen en identificaciones sexuales cruzadas. En la fase pre-edípica, entonces, en términos de Benjamin (1998), los niños son sobre-inclusivos, es decir que los niños y niñas se identifican con progenitores de ambos sexos, entonces la diferencia anatómica aún no ha operado marcando los límites de lo permitido y lo prohibido en relación con la dirección de la identificación y el deseo.

Benjamin (2002) destaca que, a diferencia de la fase sobre-inclusiva, la lógica binaria del Edipo separa tajantemente el amor de objeto -tener- y la identificación -ser-, instaurando la ficción de la inevitable exclusión entre ambos procesos. En efecto, el complejo de Edipo carga en sí el imperativo heterosexual, en donde ser y tener, identificación y amor objetal deben permanecer separados. En esta lógica de la mutua exclusión, en la que se sostiene la organización cultural heterosexual, solo es posible ser " $\mathrm{X}$ " y tener "Y" o bien ser "Y" y tener "X", identificación y amor de objeto 
están, entonces, separados. El funcionamiento escindido de ambos procesos asegura no solo la diferencia excluyente entre ambos géneros sino su relación heterosexualmente complementaria. Es así que, a partir de lo que Judith Butler (1993a) ha dado en llamar como Matriz de Inteligibilidad Heterosexual, la conformación de la identidad de género masculina requiere no solo la identificación con lo masculino, sino la elección de objeto femenino. La identificación a la base de cada uno de los géneros requiere de la dirección específica de deseo que le corresponde.

En suma, Benjamin (1997) destaca la especificidad de lo preedípico para cuestionar el ordenamiento actual de los géneros imperante en nuestro sistema cultural. La intensa fase sobre-inclusiva por la que transcurren los/as niños/as, en la cual se identifican con adultos de ambos sexos, se opone a la lógica edípica heterosexual. Durante la fase preedípica, el reconocimiento de la subjetividad materna aún era posible, pues solo posteriormente las exigencias edípicas obligan a los varones a desidentificarse de la madre y repudiar sus aspectos femeninos como referentes identificatorios. La femineidad constituye una diferencia radical a la cual solo se puede acceder vía elección de objeto y no mediante la identificación. Repudio de la madre, identificación con el padre, constitución de la identidad de género masculina, elección de mujeres como objetos sexuales son procesos simultáneos que responden a las exigencias necesarias para la emergencia de un sujeto cifrado en clave fálica a partir de la lógica edípica que opera mediante la separación entre amor objetal e identificación.

El niño resuelve la ambivalencia inherente a todo ser humano-léase bisexualidad constitutiva en Freud, o fase sobre-inclusiva en Benjaminnegando su primer vínculo significativo, mediante la proyección de los aspectos negativos sobre la figura materna y mediante la dominación de temores a la regresión o a la reidentificación con la madre mediante su control y devaluación. Estas defensas se ponen en marcha para distanciarse y controlar al objeto peligroso, luego quitarle su poder, y así mantener "la diferencia" lejos de los destinos identificatorios que contaminan la coherencia identitaria y cuestionan sus límites. 
La melancolía de género en el pensamiento de Judith Butler

En El yo y el ello, Freud (1923) conceptualiza a la melancolía como un proceso a través del cual el carácter del yo adviene a partir de las cargas libidinales de objetos abandonados que, vía identificación, son internalizadas. El yo se constituye a partir de una sedimentación de los objetos amados y luego perdidos. Paradójicamente, la identificación que opera en la melancolía preserva fantasmáticamente el objeto perdido en la esfera psíquica. Dicha incorporación, entonces, se constituye en la condición previa que permite al yo desligarse del objeto perdido. En este sentido, siguiendo a Freud, el objeto perdido recae sobre el yo, en cada una de las pérdidas, a modo de una de sus identificaciones constitutivas.

En esta línea, es posible afirmar que si el objeto es preservado en el yo, de modo que inevitablemente forma parte de él, entonces la pérdida no es total. Tal como señala Tammy Clewell (2004), se produce un trabajo de sobreinvestidura que mantiene, al menos momentáneamente, la existencia de lo perdido en el espacio de la psique. A criterio de Clewell se trata de la "...sustitución de una ausencia real por una presencia imaginaria" (Clewell, 2004, p. 44).

Butler (1995) ubica el proceso de identificación como un articulador nodal al momento de pensar el modo en que la norma social hegemónica constituye subjetividad. Se centra en la identificación melancólica y la ubica como aquel proceso privilegiado a través del cual el yo asume un carácter de género al tiempo que se constituye como tal.

Butler (1990a, 1993a, 1995) inaugura la posibilidad de pensar la formación melancólica del género. Argumenta que la identidad de género es producto de la identificación melancólica. A su criterio, en el marco de las culturas heteronormativas, el género y la melancolía producen anudamientos específicos, pues los dispositivos de control montados para mantener la heterosexualidad obligatoria (Rich, 1980) prohíben de entrada la elección de objeto del mismo sexo como posibilidad legítima. De este modo, el vínculo homosexual recae indefectiblemente sobre el yo, vía identificación melancólica, habitándolo como una de sus identificaciones constitutivas. El vínculo resignado no es abolido, sino preservado en la 
psique a través de esta internalización, que paradójicamente forma parte del mecanismo de su rechazo. Es así que la particular dinámica de la melancolía niega y preserva, de manera simultánea, la homosexualidad en la producción del género dentro del marco heterosexual. La identificación adviene en lugar de una relación con el objeto del mismo sexo, por lo tanto identificarse con uno de los géneros supone el funcionamiento melancólico que interioriza al objeto prohibido.

Si la prohibición cultural de la homosexualidad es, no obstante, incorporada y preservada ineludiblemente por vía melancólica como mecanismo del rechazo de tal opción prohibida, el vínculo homosexual prohibido no se pierde completamente. Aquello prohibido continúa operando de manera eficaz, por tanto los dispositivos de sujeción/ subjetivación encuentran sus fallas, la ausencia es presencia, los silencios gritan, la homosexualidad renegada se filtra entre los intersticios y las fisuras de un sistema heteropatriarcal que contrarresta tal amenaza con dispositivos de desigualación (Fernández, 2009).

En sintonía con los aportes de Benjamin, Butler (1993a, 2001) denuncia al modelo de subjetivación edípico como dispositivo de sujeción de la cultura heterosexual, como productor de una identidad masculina que no solo es rígida, coherente y delimitada de modo tal que excluye aspectos que recaen bajo el signo de la diferencia desigualada y repudiada, sino también produce una identidad signada por un mandato que le es inherente: el cumplimiento y la realización de la heterosexualidad obligatoria. La posición de género masculina, fijada a través de la producción de tal identidad, exige, entonces, el logro de la heterosexualidad. Sin embargo, tal logro depende de la prohibición de ciertos vínculos (homo) sexuales que, en tanto perdidos, son recuperados en la esfera psíquica como fantasmas constitutivos de la subjetividad (Bleichmar, 1992, 2006) que amenazan de manera constante la heterosexualidad.

Para Butler (1990a), los géneros no son una disposición que condiciona el devenir subjetivo de una vez y para siempre. Lejos de esta concepción, la autora permite pensar la masculinidad y la feminidad en términos de posiciones que emergen como un logro de manera conjunta con la consecución de una heterosexualidad siempre precaria, construida 
sobre una prohibición continua que implica el repudio de un segmento de la propia vida fantasmática al acatar las normas de género (Butler, 2004). El ordenamiento dicotómico de género, que el Edipo reproduce, se alcanza y se estabiliza mediante el posicionamiento heterosexual. Desde esta perspectiva, queda claro que la identidad de género funciona como virtualmente normativa, regulativa y con fuertes consecuencias políticas, opera como un sistema de control y regulación de las subjetividades de manera que los individuos responden a los patrones establecidos (Femenías, 2003). En estos términos, las identidades de género son entidades plenas, distintas y opuestas entre sí, ajustadas a modelos culturales que tienen por función separar a los sexos y establecer el privilegio absoluto de uno sobre el otro.

Sin embargo, Butler y otras pensadoras preocupadas por las vinculaciones entre los géneros y las identidades sexuales (Benjamin, 1997; Chodorow, 1978; Rubin, 1975) dejan en claro que existen múltiples formas de vivir el género y la sexualidad más allá de los límites de la lógica edípica que estabiliza los géneros a través de la complementariedad heterosexual.

No sorprende que, en el marco de una cultura heteronormativa, los sujetos elijan vivir en, o actuar la, opción heterosexual debido al miedo a la castración, es decir el temor a la feminización -fuertemente asociada a la homosexualidad masculina en las culturas heterosexuales-. En efecto, a criterio de Butler (1993a), el fantasma normativo de una heterosexualidad obligatoria-que opera a través de la naturalización y la reificación de normas heterosexistas- sobrevuela las conceptualizaciones freudianas que intentan dar cuenta de la identificación y de la dirección del deseo. Valiéndose de la categoría de restricciones constitutivas, Butler (1993a, 1995) reflexiona acerca de las formas en que la sexualidad está construida en virtud de demandas simbólicas apremiantes y constitutivas profundamente instaladas. Es así que pensar en términos de demandas simbólicas de asumir una determinada posición sexuada conduce, junto a Butler, al intento de comprender la complejidad de la trama que entreteje el ámbito en el que se imponen las restricciones sobre el sexo y la 
sexualidad -en otras palabras, los límites de lo que puede y de lo que no puede construirse-. La autora devela las bases de una heterosexualidad normativa no examinada. A partir de allí, visibiliza el modo en que a la articulación entre complejo de castración y complejo de Edipo subyacen tanto una demanda simbólica como una amenaza de castigo, como las dos caras de una misma moneda, que determinan la constitución de una fuerza obligatoria que fija los límites de un desarrollo normal -vale decir una posición heterosexual-, y más allá del cual se ubican las posiciones rechazadas como abyectas - vale decir las posiciones de gay y lesbiana, entre muchas otras (Martínez, 2009)-.

Es así que Butler (1993a) recurre al relato freudiano que explica el modo en que el complejo de Edipo y el miedo a la castración son las estructuras principales que ordenan la identificación y la dirección del deseo. El recurso a la teoría psicoanalítica le permite argumentar que la homosexualidad, en términos de identificación melancólica dirigida hacia el mismo sexo, precede y produce a la heterosexualidad. A partir de esta perspectiva, el concepto de identificación nos permite reflexionar acerca de las condiciones en las que se asumen las posiciones genéricas y sexuadas (Frosh, 1997). Asumir dichas posiciones supone, para Butler (1990a, 1995), identificarse con una ubicación dentro de la esfera simbólica, en relación con los esquemas reguladores. Para la autora, identificarse implica imaginar la posibilidad de aproximarse a dichos esquemas, a través de los cuales la imposición heterosexista opera mediante la regulación de la identificación fantasmática. En suma, un supuesto heteronormativo poco examinado atraviesa el modelo edípico freudiano, el cual presupone un deseo entre sexos opuestos. En ese contexto, Butler (1990a, 1993a, 1995) toma las ideas de Freud en relación al funcionamiento de la melancolía para afirmar el lugar central del proceso identificatorio en la producción subjetiva del género. Al mismo tiempo, dicha producción, supone siempre una pérdida ya que el objeto culturalmente idealizado no puede ser incorporado plenamente en el sujeto. En efecto, tal como ha señalado Benjamin (1997), como producto esperable del modo en que opera la dinámica del Edipo emerge un sujeto heterosexualizado identificado con la figura parental de su misma morfología corporal, el cual toma como objeto de amor a la figura parental de diferente sexo. 
Como ya se ha señalado, Butler (1990a) enlaza la incorporación de aquello perdido, por un lado, y el género, por otro, a través de la dimensión del tabú. Freud deja en claro el modo en que la prohibición del incesto ordena la intensidad de las identificaciones y del deseo al interior del complejo de Edipo. En esta línea, la autora menciona que al perder a la madre como objeto de amor, el niño incorpora tal pérdida identificándose con ella. Sin embargo, tal como afirman Chodorow (1978) y Benjamin (1996), para constituir su masculinidad, el niño debe rechazar este segmento que, vía identificación, ha constituido su propia identidad. La masculinidad hegemónica, entonces, está signada por un rechazo a sí misma. En este sentido, la identificación que constituye la identidad de género masculina requiere tanto la exclusión de las mujeres como el rechazo de cualquier homoerotismo que pueda amenazar la masculinidad culturalmente idealizada. Como afirma Butler (1995), esta identidad masculina heterosexual claramente se cultiva a través de prohibición de las posibilidades homoeróticas. Entonces, en un mundo en que la organización dicotómica, rígida y excluyente de los géneros es subsidiaria a una Matriz de Inteligibilidad Heterosexual (Butler, 1993b), los varones que portan identidades masculinas hegemónicas no pueden vincularse sexual o amorosamente con otros varones. Como el vínculo homosexual también se encuentra prohibido, para el varón, la elección de objeto heterosexual exogámica es, entonces, una formación de compromiso. En el mismo movimiento evade a la mujer concreta prohibida por el tabú del incesto -la madre- y toma como elección de objeto sexual otra/s mujer/es -todas son iguales a los fines de acatar la prohibición de la homosexualidad-. Dos prohibiciones se anudan en la identidad masculina hegemónica. Los varones tramitan y resuelven, con diferentes niveles de magnitud, sus temores, sus posibilidades de vida prohibidas no llevadas a cabo y sus pérdidas no lloradas (Butler, 2006) al interior del vínculo heterosexual.

Los límites rígidos de la organización de los géneros ocultan la pérdida de un objeto y de una modalidad de amor -a criterio de Butler, originales-, que al ser prohibidos culturalmente nunca terminan por ser aceptados ni resueltos. Como la identificación no sustituye por completo 
el objeto perdido, los efectos originados por la pérdida no pueden ser eliminados completamente. Es posible pensar que la experiencia directa de la investidura de objeto difiere de la experiencia llevada a cabo desde la identificación, pues el objeto real es inaccesible para la identificación. La sustitución absoluta es imposible y la identificación melancólica da cuenta de la problemática implicada en la constitución identitaria a causa de esta incorporación limitada. Entonces, la pérdida conforma una parte constitutiva de la identidad que guarda en sí un conflicto que persiste, sin poder ser resuelto completamente, y amenaza su coherencia interna.

Gayle Rubin (1975) ya ha mencionado el modo en que la prohibición del incesto presupone la prohibición, más sutil y solapada, de la homosexualidad. Butler (1995) sugiere que las formas culturales de vida, interpretadas desde una matriz de inteligibilidad heterosexual, se encuentran impregnadas de una ansiedad de género continua, más específicamente de un miedo al deseo homosexual que infunde pánico a mujeres y varones, por temor a la pérdida inminente de la feminidad y de la masculinidad respectivamente. Los fantasmas homosexuales incorporados melancólicamente retornan y activan la amplia gama de aspiraciones libidinales cercenadas desde los mandatos heteronormativos. El cuestionamiento de la complementariedad heterosexual se torna insoportable, pues la emergencia de fantasmas homosexuales desdibuja aquello que el Edipo ha separado tajantemente: la identificación y la dirección del deseo.

En síntesis, es posible afirmar que somos lo que no podemos tener a causa de prohibiciones culturales. Tal como afirma Jay (2007), en sintonía con Butler, feminidad y masculinidad constituyen la vestimenta funeral que utilizamos en homenaje a nuestras posibilidades homoeróticas perdidas.

\section{Conclusión y discusión}

Si bien el psicoanálisis freudiano no utiliza explícitamente los conceptos de género e identidad, al menos en el sentido que se le da en este contexto, los aportes de Benjamin y Butler abren una vía de acceso a 
una amplia gama de sentidos que giran en torno a estas categorías. Ambas autoras permiten pensar la constitución subjetiva del género a partir de la identificación. Butler (1993a, 1993b, 1995) ofrece elementos filosóficos que permiten una mayor comprensión del modo en que el niño se enfrenta con contradicciones que amenazan su propia configuración identitaria. Se trata de un choque, mencionado anteriormente, entre, por un lado, los mandatos culturales de la heterosexualidad obligatoria-como premisa definitoria de la masculinidad que exigen que el varón tome como objeto de amor a mujeres- y, por otro, la necesidad de repudiar y denigrar la feminidad amenazante para mantener los límites rígidos de la identidad masculina hegemónica.

Es necesario seguir explorando la producción del género en vinculación con la elección sexual de objeto en el contexto de posibilidades eróticas perdidas. Tanto a criterio de Butler (1993a, 1995, 2004) como de Benjamin (1996, 1997), las restricciones culturales que limitan la masculinidad y la feminidad como entidades diferenciales definen, al mismo tiempo, aquello que se pierde al quedar excluido en la construcción de uno u otro género.

En repetidas ocasiones Freud (1905) enfatiza las modalidades infantiles de satisfacción no diferenciadas y la gama de placeres difusos que se restringen de acuerdo con las exigencias de la sociedad. A pesar de ello, Butler (1990a) presta demasiada atención a la dimensión cultural del género, en la que solo existen dos opciones disponibles como referentes identificatorios. Si Butler pusiera mayor énfasis en el relato freudiano de la sexualidad infantil que en las exigencias de las identidades culturalmente hegemónicas, podría situar a la identificación como un mecanismo que guarda gran potencialidad a la hora de pensar nuevas formas de ser que van más allá de aquellas culturalmente impuestas. En su intento de reivindicar la homosexualidad como opción legítima, Butler (1990a, 1993b, 1995) restringe su narrativa a solo dos destinos de la identificación, que aseguran a priori el anclaje subjetivo de las identidades de género culturalmente ofrecidas. ¿Por qué restringir el debate a los términos de varón, mujer, homosexual y heterosexual, como si estas fueran las únicas opciones posibles? 
En esta línea, si no perdemos de vista el carácter polimorfo de la sexualidad, las restricciones que ponen en marcha la identificación melancólica son mucho más amplias que la homosexualidad. Por otra parte, si bien es posible pensar que cualquier identificación constitutiva de las identidades de género implica al mismo tiempo el fracaso de una gama de placeres, tampoco debemos perder de vista que en el marco de una cultura heteronormativa dominante algunas pérdidas cuentan más que otras. Aún así, si enfatizamos el funcionamiento del mecanismo de la identificación en sentido estricto, tanto la heterosexualidad como la homosexualidad son melancólicas, pues cualquier restricción de placeres implica una pérdida. Es decir, si somos, como afirma Freud, perversos polimorfos, la construcción subjetiva del género, varón o mujer, y de la sexualidad, homosexual o heterosexual, necesariamente implica renunciar a elementos que dan lugar a tales identificaciones.

Claramente, Butler (1990a) se propone derribar la idea de identidades de género hegemónicamente delimitadas como normales. Basándose en conceptualizaciones freudianas propone una interpretación del género en tanto resultado precario, logrado a partir de una exclusión continua que se realiza a un costo significativo. Si bien el proceso de identificación restringe los placeres polimorfos propios de la sexualidad infantil, inscribir tal restricción en los términos del binario heterosexualidad/ homosexualidad supone introducir -a priori- en los argumentos una gama de prohibiciones que impiden pensar otras formas de género más allá del binario varón/mujer.

Los aportes de Butler no dejan de ser importantes a la hora de desenmascarar el sistema heteronormativo como productor de deseos naturalizados. Sin embargo, al centrarse exclusivamente en la homosexualidad, no lleva hasta sus últimas consecuencias la potencialidad de sus propias ideas.

Resulta valioso pensar la producción de género en términos de identificación y de elección de objeto, y no como una condición ontológica del ser. La categoría de identificación devela el modo en que se constituye la identidad, al mismo tiempo que tal mecanismo introduce 
aquello a lo que el sujeto debe renunciar para sostener la exigencia cultural de coherencia. Es así que la concepción freudiana de identificación melancólica da cuenta del complejo giro mediante el cual se constituye la identidad de género, culturalmente ofrecida, a nivel subjetivo. Las exigencias culturales imponen auto-renuncias de otras posibilidades de habitar el género y la sexualidad.

La originalidad de Butler a la hora de plantear la identidad de género como producto de la interiorización eficaz del tabú de la homosexualidad es notable. Situar el mecanismo de la melancolía como respuesta subjetiva ante la pérdida del objeto del mismo sexo en los primeros tiempos de la vida psíquica, y a la vez anudar tal pérdida a una prohibición sostenida por la contingencia de una norma social, supone transversalizar el psicoanálisis con una dimensión histórico-social y política poco tenida en cuenta. De este modo, Butler logra conectar aquellos mecanismos de funcionamiento psíquico con determinaciones que exceden las coordenadas individuales. Sus aportes permiten poner a funcionar la potencialidad explicativa de la teoría psicoanalítica a la hora de explicar, en su mayor complejidad posible, la constitución subjetiva del género sin dejar a un lado las tensiones de poder que circulan entre sus conceptos.

\section{Referencias}

Aulagnier, P. (2004). La violencia de la interpretación (V. Fischman trad.). Buenos Aires: Amorrortu. (Trabajo original publicado en 1975).

Benjamin, J. (1996). Los lazos de amor. Psicoanálisis, feminismo y el problema de la dominación (J. Piatigorsky trad.). Buenos Aires: Paidós. (Trabajo original publicado en 1988).

Benjamin, J. (1997). Sujetos iguales, Objetos de amor. Ensayos sobre el reconocimiento y la diferencia sexual (J. Piatigorsky trad.). Buenos Aires: Paidós. (Trabajo original publicado en 1995).

Benjamin, J. (1998). Shadow of the Other. Intersubjectivity and Gender in Psychoanalysis. New York/London: Routledge.

Benjamin, J. (2002). The Question of Sexual Difference. Feminism \& Psychology, 12(1), 39-43. 
Bleichmar, S. (1992). Paradojas de la constitución sexual masculina. Revista Asociación Escuela Argentina de Psicoterapia para Graduados, 18, 175-188.

Bleichmar, S. (1995). Las condiciones de la identificación. Revista Asociación Escuela Argentina de Psicoterapia para Graduados, 21, 201-219.

Bleichmar, S. (2006). Las Paradojas de la Sexualidad Masculina. Buenos Aires: Paidós.

Burin, M. \& Meler, I. (1998). Género y familia. Poder, amor y sexualidad en la construcción de la subjetividad. Buenos Aires: Paidós.

Butler, J. (1990a). Gender Trouble. Feminism and the Subversion of Identity. New York: Routledge.

Butler, J. (1990b). Performative Acts and Gender Constitution: An Essay in Phenomenology and Feminist Theory. En Sue-Ellen Case (ed.). Performing Feminisms: Feminist Critical Theory and Theatre (pp.270282). Baltimore: Johns Hopkins University Press.

Butler, J. (1993a). Bodies that matter. New York: Routledge.

Butler, J. (1993b). Imitation and Gender Insubordination. En H. Abelove, M. A. Barale \& D. Halperin (eds.). The Lesbian and Gay Studies Reader (pp.307-320). New York: Routledge.

Butler, J. (1995). Melancholy Gender - Refused Identification. Psychoanalytic Dialogues, 5(2), 165-180.

Butler, J. (2001). Mecanismos psíquicos del poder: Teorías sobre la sujeción (J. Cruz trad.). Madrid: Cátedra. (Trabajo original publicado en 1997).

Butler, J. (2004). Undoing Gender. New York: Routledge.

Butler, J. (2006). Vida precaria. El poder del duelo y la violencia (F. Rodríguez trad.). Buenos Aires: Paidós. (Trabajo original publicado en 2004).

Campbell, J. (1997). Mediations of the female imaginary and symbolic. History of the Human Sciences, 10(2), 41-60.

Chodorow, N. (1974). Family structure and feminine personality. In M.Z. Rosaldo \& L. Lamphere (Eds.), Woman, Culture and Society. California: Stanford University Press.

Chodorow, N. (1978). The Reproduction of Mothering: Psychoanalysis and the Sociology of Gender. Berkeley: University of California Press.

Clewell, T. (2004). Mourning Beyond Melancholia: Freud's Psychoanalysis of Loss. Journal of the American Psychoanalytic Association, 52(1), 4367.

Dio Bleichmar, E. (1992). Del sexo al género. Revista Asociación Escuela Argentina de Psicoterapia para Graduados, 18, 127-155. 
Driver, S. (2005). Intersubjetive openings. Rethinking feminist psychoanalytics of desire beyond heteronormative ambivalence. Feminist Theory, 6(1), 5-24.

Fast, I. (1990). Aspects of early gender development: Toward a reformulation. Psychoanalytic Psychology, 7, 105-118.

Femenías, M. L. (2003). Judith Butler: Introducción a su lectura. Buenos Aires: Catálogos.

Femenías, M. L. (2009). Nuevas violencias contra las mujeres. Nomadias, 10, 11-28.

Fernández, A. M. (2009). Las lógicas sexuales: Amor, politica y violencias. Buenos Aires: Nueva Visión.

Flax, J. (1995). Psicoanálisis y Feminismo. Pensamientos Fragmentarios (C. Martínez Gimeno trad.). Madrid: Cátedra. (Trabajo original publicado en 1990).

Freud, S. (1989/1905). Tres ensayos para una teoría sexual. En Obras Completas, Tomo VIII, Buenos Aires: Amorrortu.

Freud, S. (1989/1921). Psicología de las masas y análisis del yo. En Obras Completas, Tomo XVIII, Buenos Aires: Amorrortu.

Freud, S. (1989/1923). El yo y el ello. En Obras Completas, Tomo XIX, Buenos Aires: Amorrortu.

Frosh, S. (1997). Psychoanalytic Challenges: a contribution to the new sexual agenda. Human Relations, 50(3), 229-239.

Gergen, M. (1992). Undbundling Our Binaries - Genders, Sexualities, Desires. Feminism \& Psychology, 2(3), 447-450.

Jay, M. (2007). Individual Differences in Melancholy Gender Among Women: Does Ambivalence Matter? Journal of the American Psychoanalytic Association, 55(4), 1279-1320.

Kristeva, J. (2009). Esa increíble necesidad de creer (V. Ackerman \& S. Mattoni trads.). Buenos Aires: Paidós. (Trabajo original publicado en 2006).

Lamas, M. (1986). La antropología feminista y la categoría de 'género'. Nueva Antropología, 8, 30, 173-198.

Martínez, A. (2009). (Hetero)Sexualidad e Identidad de Género. Aportes del Feminismo y los Estudios de Género al Psicoanálisis Contemporáneo. Revista Psico-Logos, 19, 11-26.

Reinharz, S. (1992). How My Heterosexuality Contributes to My Feminism and Vice Versa. Feminism \& Psychology, 2(3), 450-453.

Rich, A. (1980). Compulsory Heterosexuality and Lesbian Existence. Signs, 4(5), 631-660. 
Rich, A. (1986) Nacemos de mujer. La maternidad como experiencia y como institución (A. Becciu trad.). Madrid: Cátedra. (Trabajo original publicado en 1976).

Rubin, G. (1975). The Traffic in Women: Notes on the 'Political Economy' of Sex. En Rayna R. Reiter (ed.), Toward an Anthropology of Women, Nueva York: Monthly Review Press.

Segal, L. (2008). Los hombres tras el feminismo: ¿Qué queda por decir? En À. Carabí \& J. M. Armengol (eds.). La masculinidad a debate (pp.155-175). Barcelona: Icaria.

Silverman, K. (1988). The Acoustic Mirror. Bloomington: Indiana.

Spillers, H. (1987). Mama's baby, Papa’s maybe: An American Grammar Book. Diacritics, 17(2), 65-81.

Stoller, R. (1968). Sex and Gender. New York: Science House.

Thompson, D. (1992). Against the Dividing of Woman: Lesbian feminism and Heterosexuality. Feminism and Psychology, 2(3), 387-398.

Tort, M. (2008). Fin del dogma Paterno (V. Ackerman trad.). Buenos Aires: Paidós. (Trabajo original publicado en 2005).

Weir, A. (1992). The paradox of the self: Jessica Benjamin's intersubjetive theory. Thesis Eleven, 32, 141-153.

Young, A. (1992). The Authority of the Name. Feminism \& Psychology, 2(3), 422-424.

Fecha de recepción: 25 de agosto de 2010.

Fecha de aceptación: 10 de diciembre de 2010. 